Masaki Mizutani - Toshimichi Yamamoto $\cdot$ Kouji Torii

Hirokazu Kawase • Takashi Yoshimoto • Rieko Uchihi

Miwa Tanaka - Keiji Tamaki • Yoshinao Katsumata

\title{
Analysis of 168 short tandem repeat loci in the Japanese population, using a screening set for human genetic mapping
}

Received: March 26, 2001 / Accepted: April 25, 2001

\begin{abstract}
We devised a multiplex polymerase chain reaction (PCR) amplification and loading system for the convenient typing of 168 short tandem repeat (STR) polymorphic markers in a commercially available screening primer set for human linkage analysis. We genotyped all these 168 STR loci with 32 healthy unrelated Japanese, calculated allele frequencies at each STR locus, and performed three kinds of tests for Hardy-Weinberg equilibrium (HWE). Significant deviations from HWE in all three tests were observed at only three loci, and the average heterozygosity in the Japanese (0.733) was slightly lower than that in Caucasians $(0.773)$. We also examined 32 Caucasians at some selected loci, to be compared with Japanese. Some markers showed greatly different heterozygosities or allelic distributions in Japanese and Caucasian populations. In two groups of STRs, those with and without irregular alleles (or interalleles), the former had a higher proportion of bimodal allelic distribution and possessed more alleles per locus than the latter. However, no significant differences in the observed and expected heterozygosities, or in the powers of discrimination, were found between the two groups. The present basic study of allele frequency databases of these STRs will contribute to further applications in forensic science and human genetics.
\end{abstract}

Key words Short tandem repeat - Polymorphism . Multiplex · Heterozygosity · Allele frequency · Japanese · Population

M. Mizutani · T. Yamamoto $(\bowtie) \cdot$ K. Torii $\cdot$ H. Kawase

T. Yoshimoto $\cdot$ R. Uchihi - M. Tanaka $\cdot$ Y. Katsumata

Department of Legal Medicine and Bioethics, Graduate School of

Medicine, Nagoya University, 65 Tsurumai-cho, Showa-ku, Nagoya 466-8550, Japan

Tel. +81-52-744-2119; Fax +81-52-744-2121

e-mail: yamachan@med.nagoya-u.ac.jp

K. Tamaki

Department of Legal Medicine, Sapporo Medical University, School of Medicine, Sapporo, Japan

\section{Introduction}

Short tandem repeat (STR) polymorphisms are widely used in various scientific fields, such as linkage analyses and diversity studies in human genetics, and personal identification and paternity tests in forensic science (Wijmenga et al. 1990; Schellenberg et al. 1992; Bowcock et al. 1994; Micka et al. 1996; Thomson et al. 1999), because they have relatively high heterozygosities and are more easily genotyped than other chromosomal markers. More than one million STRs (one $/ 2 \mathrm{~kb}$ ) are widely distributed in the whole human genome (International Human Genome Sequencing Consortium 2001), and most of them consist of dinucleotide repeats, in particular, CA repeats (Gyapay et al. 1994). Although these dinucleotide STRs are most commonly used for genetic linkage mapping, there are some problems in genotyping, caused by their artifactual "stutter" bands, particularly in the sizing of heterozygous samples and/or mixed samples. Therefore, tetranucleotide STRs showing smaller "stutter" bands are mainly used in forensic practice (Micka et al. 1996; Thomson et al. 1999; Yamamoto et al. 1999; Sweet and Hildebrand 1999).

Dubovsky et al. created a set of STRs for the genetic linkage mapping of disease loci by combining highly polymorphic STRs that are distributed evenly throughout the human genome (Dubovsky et al. 1995). The first version of this set included a high percentage (49\%) of dinucleotide STRs. The latest version of the screening set, the Human MapPair screening set 8A, contains 168 STR markers, spaced at an average genetic distance of $25 \mathrm{cM}$ throughout the entire human genome. Most of the dinucleotide STRs were replaced by tetranucleotide STRs in this set, and this set consists of a high percentage $(83 \%)$ of tetranucleotide STRs, allowing greater accuracy in the determination of their repeat sizes.

We investigated allele frequency distributions and heterozygosities at all 168 STR markers in the screening set $8 \mathrm{~A}$ in the Japanese population. The results could be useful for the genetic mapping of human diseases, for forensic 
applications, and for studies of population genetics, such as the origin of the Japanese.

\section{Materials and methods}

Blood samples were obtained, with written informed consent, from 32 healthy unrelated Japanese volunteers (undergraduate students at our medical school who lived in or near Nagoya city). DNA was extracted from each sample as described previously (Tamaki et al. 1991). The 168 markers were genotyped using each primer set in the screening set $8 \mathrm{~A}$ (Research Genetics, Huntsville, AL, USA), each one of which is labeled with any one of three different fluorescentcolored dyes (FAM, HEX, and TET). We divided these primer sets into groups that could be amplified in a single PCR tube (Table 1), and then performed multiplex PCR amplification. The PCR mixture $(10 \mu \mathrm{l})$ contained $1 \times \mathrm{PCR}$ Gold Buffer, $1.5 \mathrm{mM} \mathrm{MgCl}_{2}, 200 \mu \mathrm{M}$ of each dNTP, $2.4 \mathrm{pmol}$ of each STR primer set, 0.25 unit AmpliTaq Gold polymerase (Perkin-Elmer, Norwalk, CT, USA), and 10ng of template DNA. Thermal cycling protocols began with $95^{\circ} \mathrm{C}$ for $11 \mathrm{~min}$ and then 26 cycles at $94^{\circ} \mathrm{C}$ for $45 \mathrm{~s}, 57^{\circ} \mathrm{C}$ for $45 \mathrm{~s}$, and $72^{\circ} \mathrm{C}$ for $60 \mathrm{~s}$, followed by $72^{\circ} \mathrm{C}$ for $15 \mathrm{~min}$. One $\mu \mathrm{l}$ of each of the PCR set products was mixed into $24.5 \mu$ of deionized formamide and $0.5 \mu$ l of GeneScan-500 [TAMRA] Size Standard (PE Applied Biosystems, Foster City, CA, USA), according to the loading panel shown in Table 1. For example, for loading panel $1,1 \mu \mathrm{l}$ of each of the PCR set 1,2,3, and 4 products was mixed together into the formamide and standard mixture. Allels were separated in the POP-4 polymer (PE Applied Biosystems) with the GS STR POP 4 (1 mL) C Module, by capillary electrophoresis, using the Genetic Analyzer 310 (PE Applied Biosystems). Allele sizes for each STR were determined on the basis of sizing data analyzed by GeneScan Analysis software (PE Applied Biosystems). For some representative loci, we also examined 64 Caucasian chromosomes to calculate allele frequencies at each locus, and compared each allele frequency in Japanese and Caucasians, using the software GENEPOP 3.1b (Raymond and Rousset 1995). The Caucasian DNA samples (from the United Kingdom) were kindly provided by Dr. Yuri E. Dubrova, at the University of Leicester.

Tests for Hardy-Weinberg equilibrium (HWE) were carried out using a homozygosity test (Weir 1992), a likelihood ratio test (Chakraborty et al. 1991), and an exact test (Guo and Thompson 1992). As statistical properties, the observed heterozygosity, the unbiased estimates of expected heterozygosity (Edwards et al. 1992), and the power of discrimination (Fisher 1951) were calculated. Heterozygosities in Caucasians were obtained from the Research Genetics website (http://www.resgen.com), which does not give details about the source of these samples. The sequence data for each STR were obtained from the Genome Data base (GDB; http://gdbwww.gdb.org), the Cooperative Human Linkage Center (CHLC; http://lpg.nci.nih.gov/CHLC) and other organizations, and served for our reference to determine sizes at each STR locus.

\section{Results and discussion}

The 168 STR markers were grouped into 103 temporary multiplex PCR sets for genotyping by multiloading performed 21 times (Table 1). From the result of sizing, we temporarily named the shortest allele observed in the Japanese population at each locus as allele 1 , and the larger alleles following at every repeat as alleles $2,3,4,5, \ldots$ in order, taking into consideration the sequence data from the websites noted above. The irregular alleles observed were named according to an established recommendation (Gill et al. 1997). Using this temporary nomenclature for alleles, we genotyped and calculated allele frequencies at each locus. From these frequencies, three kinds of tests (the homozygosity test, likelihood ratio test, exact test) were performed to check deviation from HWE. The allele frequencies deviated from HWE ( $P$ values fell to less than 0.05) for 8 loci in the homozygosity test, 11 loci in the likelihood ratio test, and 13 loci in the exact test. However, it was only at 3 loci (D7S3051, D19S586, and D1S1588) that significant deviations from HWE were observed with all three tests. Furthermore, of the 8 loci whose $P$ values were less than 0.05 in the homozygosity test, 5 showed very low observed heterozygosities $(<0.60)$, resulting in large differences between the observed and expected heterozygosities. These results may have been caused by a contingent event, sampling error, or population substructure. An investigation using more samples would, presumably, explain this result.

Table 1 summarizes the size ranges, numbers of alleles, statistical properties of the 168 markers in the Japanese population, and the observed heterozygosity in Caucasians obtained from the Research Genetics website. The observed heterozygosity at 158 autosomal STRs ranged from 0.38 to 0.94 in the Japanese population, varying more widely than among Caucasians. The mean observed heterozygosity was 0.733 , slightly below that in Caucasians (0.773). In some markers, the heterozygosity in the Japanese population was very different from that in the Caucasian population. For example, heterozygosities at D1S1588 and D18S481 were 0.38 and 0.91 in Japanese, against 0.68 and 0.76, respectively, in Caucasians. Spearman's correlation coefficient (Siegel 1956) between Japanese and Caucasians was 0.372 , indicating considerably different allele distributions in the two populations. These results suggest the necessity of making a database for each population. We also calculated the expected heterozygosity at each locus, and found a mean value of 0.747 . The variance of the expected heterozygosities (SD, 0.086) was significantly smaller than that of the observed heterozygosities (SD, 0.111 ). Figure 1 summarizes the distribution of the observed and expected heterozygosities in Japanese and the observed heterozygosity in Caucasians. Comparison of the Japanese allelic distributions with those of Caucasians at some representative loci showed a significant difference $(P<0.05)$ at almost all loci, using the software GENEPOP 3.1b (examples are shown in Fig. 2). Two alleles (alleles 1 and 2) of the D7S1823 locus are much more frequent in Japanese 
Table 1. Multiplex PCR sets and statistical properties at each locus in the Japanese population

\begin{tabular}{|c|c|c|c|c|c|c|c|c|c|c|c|}
\hline $\begin{array}{l}\text { Loading } \\
\text { Panel }\end{array}$ & $\begin{array}{l}\text { PCR } \\
\text { set }\end{array}$ & Locus & Marker & $\begin{array}{l}\text { Repeat } \\
\text { unit }\end{array}$ & $\begin{array}{l}\text { Irregular } \\
\text { allele }\end{array}$ & $\begin{array}{l}\text { Size } \\
\text { range }\end{array}$ & Allele & PD & $\begin{array}{l}\text { Expected } \\
\text { H. }\end{array}$ & $\begin{array}{l}\text { Observed } \\
\text { H. }\end{array}$ & $\begin{array}{l}\text { Observed H. } \\
\text { (Caucasian) }\end{array}$ \\
\hline \multirow{9}{*}{0} & \multirow{3}{*}{1} & D3S2418 & ATA22E01 & Tri & \multirow{9}{*}{+} & $80-110$ & 7 & 0.81 & 0.66 & 0.59 & 0.71 \\
\hline & & D4S2361 & ATA2A03 & Tri & & 149-164 & 6 & 0.87 & 0.75 & 0.75 & 0.74 \\
\hline & & D1S2134 & GATA72H07 & Tetra & & $253-289$ & 5 & 0.86 & 0.74 & 0.81 & 0.84 \\
\hline & \multirow{3}{*}{2} & D5S1456 & GATA11A11 & Tetra & & $188-208$ & 6 & 0.88 & 0.77 & 0.78 & 0.78 \\
\hline & & D6S1056 & GATA68H04 & Tetra & & $232-256$ & 7 & 0.92 & 0.85 & 0.94 & 0.85 \\
\hline & & D20S481 & GATA47F05 & Tetra & & $235-247$ & 4 & 0.81 & 0.66 & 0.66 & 0.83 \\
\hline & \multirow[t]{2}{*}{3} & D10S1239 & GATA64A09 & Tetra & & $155-179$ & 5 & 0.78 & 0.60 & 0.59 & 0.75 \\
\hline & & D3S2387 & GATA22G12 & Tetra & & $170-214$ & 11 & 0.94 & 0.86 & 0.84 & 0.86 \\
\hline & 4 & D18S481 & AFM321xc9 & $\mathrm{Di}$ & & $181-193$ & 7 & 0.87 & 0.78 & 0.91 & 0.76 \\
\hline \multirow{8}{*}{1} & \multirow[t]{2}{*}{5} & D2S1400 & GGAA20G10 & Tetra & & $108-116$ & 3 & 0.64 & 0.52 & 0.59 & 0.66 \\
\hline & & D1S1679 & GGAA5F09 & Tetra & & $143-171$ & 8 & 0.92 & 0.85 & 0.81 & 0.84 \\
\hline & \multirow[t]{2}{*}{6} & D7S3051* & GATA137H02 & Tetra & & $144-176$ & 9 & 0.89 & 0.79 & 0.59 & 0.75 \\
\hline & & D17S1308 & GTAT1A05 & Tetra & & 294-310 & 5 & 0.75 & 0.60 & 0.56 & 0.66 \\
\hline & \multirow[t]{2}{*}{7} & D7S2846 & GATA31A10 & Tetra & & 169-189 & 6 & 0.86 & 0.72 & 0.69 & 0.76 \\
\hline & & D10S677 & GGAA2F11 & Tetra & & $197-221$ & 7 & 0.93 & 0.84 & 0.78 & 0.81 \\
\hline & \multirow[t]{2}{*}{8} & D11S4464 & GATA64D03 & Tetra & & $226-250$ & 7 & 0.90 & 0.76 & 0.66 & 0.78 \\
\hline & & D1S1660 & GATA48B01 & Tetra & & $227-247$ & 6 & 0.88 & 0.81 & 0.88 & 0.78 \\
\hline \multirow{9}{*}{2} & \multirow{3}{*}{9} & D11S1999 & GATA23F06 & Tetra & & $104-132$ & 8 & 0.87 & 0.70 & 0.72 & 0.80 \\
\hline & & D14S617 & GGAA21G11 & Tetra & & $136-174$ & 7 & 0.89 & 0.76 & 0.72 & 0.78 \\
\hline & & D3S2460 & GATA68F07 & Tetra & & $154-166$ & 4 & 0.83 & 0.69 & 0.72 & 0.76 \\
\hline & \multirow{3}{*}{10} & D15S655 & ATA28G05 & Tri & & $133-148$ & 4 & 0.73 & 0.59 & 0.66 & 0.72 \\
\hline & & D7S1823 & GATA30D09 & Tetra & & $205-233$ & 8 & 0.92 & 0.82 & 0.91 & 0.85 \\
\hline & & D5S1505 & GATA62A04 & Tetra & & $246-270$ & 7 & 0.92 & 0.84 & 0.78 & 0.80 \\
\hline & \multirow[t]{2}{*}{11} & D5S2500 & GATA67D03 & Tetra & + & $151-175$ & 8 & 0.87 & 0.76 & 0.72 & 0.82 \\
\hline & & D5S820 & GATA6E05 & Tetra & & $182-206$ & 6 & 0.91 & 0.78 & 0.72 & 0.77 \\
\hline & 12 & D5S1725 & GATA89G08 & Tetra & & 184-208 & 7 & 0.88 & 0.79 & 0.75 & 0.77 \\
\hline & 13 & D16S2616 & ATA41E04 & Tri & & $114-132$ & 6 & 0.82 & 0.64 & 0.56 & 0.69 \\
\hline & & D6S474 & GATA31 & Tetra & & $149-165$ & 5 & 0.81 & 0.66 & 0.47 & 0.77 \\
\hline & 14 & D18S858 & ATA23G05 & Tri & & $190-205$ & 5 & 0.80 & 0.64 & 0.53 & 0.75 \\
\hline 3 & & D11S2000 & GATA28D01 & Tetra & + & $196-236$ & 18 & 0.95 & 0.92 & 0.84 & 0.87 \\
\hline & 15 & D18S878 & GATA7E12 & Tetra & & $158-186$ & 7 & 0.91 & 0.82 & 0.91 & 0.76 \\
\hline & & D5S2488 & ATA20G07 & Tri & & $211-238$ & 7 & 0.86 & 0.70 & 0.66 & 0.74 \\
\hline & 16 & D19S591 & GATA44F10 & Tetra & & $92-108$ & 5 & 0.81 & 0.72 & 0.72 & 0.74 \\
\hline & 17 & $\mathrm{D} 17 \mathrm{~S} 1293$ & GGAA7D11 & Tetra & + & $264-296$ & 9 & 0.93 & 0.85 & 0.84 & 0.83 \\
\hline & & DXS6789 & GATA31F01 & Tetra & & $115-147$ & 5 & & & & \\
\hline & 18 & D7S1824 & GATA32C12 & Tetra & & $162-190$ & 7 & 0.81 & 0.68 & 0.56 & 0.82 \\
\hline 4 & & & GGAAT1B07 & Penta & & 181-196 & 4 & & & & \\
\hline & & DXS9896 & GATA124E07 & Tetra & & $186-234$ & 8 & & & & \\
\hline & 19 & D22S689 & GATA21F03 & Tetra & & $203-227$ & 7 & 0.88 & 0.74 & 0.72 & 0.76 \\
\hline & & DYS389 & GATA30F10 & Tetra & & $244-256$ & 4 & & & & \\
\hline & & D22S683 & GATA11B12 & Tetra & + & $159-203$ & 14 & 0.92 & 0.82 & 0.69 & 0.90 \\
\hline & 20 & D19S246 & $\operatorname{Mfd} 232$ & Tetra & & $182-222$ & 7 & 0.88 & 0.74 & 0.78 & 0.82 \\
\hline & & D15S642 & GATA27A03 & Tetra & + & $196-212$ & 7 & 0.87 & 0.74 & 0.66 & 0.81 \\
\hline 5 & 21 & D19S254 & $\operatorname{Mfd} 238$ & Tetra & & $112-140$ & 7 & 0.91 & 0.79 & 0.72 & 0.75 \\
\hline & 22 & D9S1118 & GATA71E08 & Tetra & & $137-173$ & 8 & 0.91 & 0.82 & 0.84 & 0.81 \\
\hline & 23 & $\mathrm{D} 22 \mathrm{~S} 420$ & AFM217xf4 & Di & & $144-156$ & 7 & 0.88 & 0.75 & 0.69 & 0.77 \\
\hline & 24 & D2S1384 & GATA52A04 & Tetra & & $132-156$ & 7 & 0.86 & 0.71 & 0.78 & 0.80 \\
\hline & & D14S606 & GATA30A03 & Tetra & & $260-276$ & 5 & 0.71 & 0.48 & 0.53 & 0.73 \\
\hline & 25 & D5S807 & GATA3A04 & Tetra & & $164-204$ & 6 & 0.86 & 0.73 & 0.63 & 0.76 \\
\hline 6 & & D8S1128 & GATA21C12 & Tetra & & $230-258$ & 7 & 0.82 & 0.68 & 0.72 & 0.76 \\
\hline & 26 & D15S643 & GATA50G06 & Tetra & + & $198-224$ & 10 & 0.94 & 0.85 & 0.84 & 0.86 \\
\hline & & D9S934 & GATA64G07 & Tetra & & $202-230$ & 8 & 0.90 & 0.77 & 0.81 & 0.76 \\
\hline & 27 & D2S1399 & GGAA20G04 & Tetra & & $133-177$ & 12 & 0.94 & 0.87 & 0.88 & 0.80 \\
\hline & 28 & D2S1356 & ATA4F03 & Tri & & $222-249$ & 8 & 0.91 & 0.80 & 0.78 & 0.76 \\
\hline & 29 & D14S306 & GATA4B04 & Tetra & & $187-211$ & 7 & 0.91 & 0.79 & 0.72 & 0.79 \\
\hline & & D19S586* & GATA23B01 & Tetra & & $231-247$ & 5 & 0.65 & 0.63 & 0.88 & 0.73 \\
\hline & 30 & D1S552 & GGAT2A07 & Tetra & & $212-256$ & 5 & 0.81 & 0.66 & 0.72 & 0.72 \\
\hline & & D15S822 & GATA88H02 & Tetra & & $234-302$ & 16 & 0.94 & 0.87 & 0.88 & 0.77 \\
\hline
\end{tabular}


Table 1. Continued

\begin{tabular}{|c|c|c|c|c|c|c|c|c|c|c|c|}
\hline $\begin{array}{l}\text { Loading } \\
\text { Panel }\end{array}$ & $\begin{array}{l}\text { PCR } \\
\text { set }\end{array}$ & Locus & Marker & $\begin{array}{l}\text { Repeat } \\
\text { unit }\end{array}$ & $\begin{array}{l}\text { Irregular } \\
\text { allele }\end{array}$ & $\begin{array}{l}\text { Size } \\
\text { range }\end{array}$ & Allele & $\mathrm{PD}$ & $\begin{array}{l}\text { Expected } \\
\text { H. }\end{array}$ & $\begin{array}{l}\text { Observed } \\
\text { H. }\end{array}$ & $\begin{array}{l}\text { Observed H } \\
\text { (Caucasian) }\end{array}$ \\
\hline \multirow[t]{5}{*}{7} & 31 & D21S2052 & GATA129D11 & Tetra & \multirow{5}{*}{+} & $120-148$ & 8 & 0.91 & 0.82 & 0.84 & 0.77 \\
\hline & 32 & D14S1426 & GATA136B01 & Tetra & & $129-157$ & 11 & 0.89 & 0.79 & 0.81 & 0.78 \\
\hline & 33 & D1S1609 & GATA50F11 & Tetra & & $175-203$ & 8 & 0.88 & 0.77 & 0.72 & 0.80 \\
\hline & 34 & D18S843 & ACT1A01 & Tri & & $181-193$ & 5 & 0.83 & 0.74 & 0.84 & 0.75 \\
\hline & 35 & D20S480 & GATA45B10 & Tetra & & 278-306 & 8 & 0.92 & 0.85 & 0.91 & 0.76 \\
\hline \multirow{8}{*}{8} & & D4S1627 & GATA7D01 & Tetra & \multirow{8}{*}{+} & $176-200$ & 7 & 0.91 & 0.81 & 0.75 & 0.81 \\
\hline & 36 & F13A1 & SE30 & Tetra & & $178-188$ & 4 & 0.73 & 0.57 & 0.53 & 0.78 \\
\hline & & D1S1665 & GATA61A06 & Tetra & & $220-252$ & 7 & 0.89 & 0.77 & 0.72 & 0.74 \\
\hline & 37 & D5S2845 & GATA134B03 & Tetra & & $137-161$ & 6 & 0.89 & 0.76 & 0.69 & 0.66 \\
\hline & & D4S2639 & GATA90B10 & Tetra & & $158-190$ & 9 & 0.92 & 0.82 & 0.81 & 0.85 \\
\hline & 38 & D7S1842 & GGAA6D03 & Tetra & & $123-147$ & 7 & 0.91 & 0.79 & 0.72 & 0.83 \\
\hline & 39 & D17S1303 & GATA64B04 & Tetra & & $221-241$ & 6 & 0.88 & 0.77 & 0.81 & 0.70 \\
\hline & 40 & D20S470 & GGAA7E02 & Tetra & & $273-313$ & 9 & 0.95 & 0.89 & 0.84 & 0.87 \\
\hline \multirow{9}{*}{9} & 41 & D12S1045 & ATA29A06 & Tri & & $75-90$ & 5 & 0.86 & 0.79 & 0.94 & 0.80 \\
\hline & & D3S2432 & GATA27C08 & Tetra & & $131-155$ & 7 & 0.90 & 0.79 & 0.72 & 0.83 \\
\hline & 42 & D21S1432 & GATA11C12 & Tetra & & $129-145$ & 5 & 0.83 & 0.71 & 0.75 & 0.63 \\
\hline & & D1S1597 & GATA27E01 & Tetra & & $156-176$ & 6 & 0.87 & 0.75 & 0.78 & 0.71 \\
\hline & 43 & D16S753 & GGAA3G05 & Tetra & & 149-169 & 6 & 0.90 & 0.78 & 0.72 & 0.79 \\
\hline & & D11S1984 & GGAA17G05 & Tetra & & $179-203$ & 7 & 0.91 & 0.82 & 0.78 & 0.79 \\
\hline & 44 & D2S1391 & GATA65C03 & Tetra & & $117-129$ & 4 & 0.76 & 0.63 & 0.72 & 0.79 \\
\hline & 45 & D11S1392 & GATA6B09 & Tetra & & $188-216$ & 7 & 0.86 & 0.71 & 0.66 & 0.77 \\
\hline & 46 & D11S2359 & ATA27C09 & Tri & + & $210-229$ & 7 & 0.88 & 0.76 & 0.69 & 0.73 \\
\hline \multirow{4}{*}{10} & 47 & D2S1394 & GATA69E12 & Tetra & & $159-175$ & 5 & 0.85 & 0.71 & 0.72 & 0.70 \\
\hline & & D5S1470 & GATA7C06 & Tetra & & $163-203$ & 10 & 0.92 & 0.81 & 0.81 & 0.82 \\
\hline & 48 & D11S2371 & GATA90D07 & Tetra & & $173-205$ & 6 & 0.87 & 0.75 & 0.78 & 0.67 \\
\hline & 49 & D7S2212 & GATA87D11 & Tetra & & $187-203$ & 5 & 0.77 & 0.58 & 0.63 & 0.73 \\
\hline \multirow{7}{*}{11} & 50 & D13S285 & AFM309va9 & Di & & 80-106 & 12 & 0.94 & 0.85 & 0.81 & 0.81 \\
\hline & & D20S171 & AFM046xf6 & $\mathrm{Di}$ & & $128-140$ & 7 & 0.87 & 0.78 & 0.88 & 0.78 \\
\hline & 51 & ACTC & ACTC & $\mathrm{Di}$ & & $64-90$ & 9 & 0.93 & 0.81 & 0.78 & 0.87 \\
\hline & & D9S158 & AFM073yb11 & $\mathrm{Di}$ & & $213-225$ & 7 & 0.83 & 0.74 & 0.81 & 0.69 \\
\hline & 52 & D8S264 & $143 x d 8$ & $\mathrm{Di}$ & & $121-141$ & 10 & 0.93 & 0.84 & 0.78 & 0.83 \\
\hline & & D3S2427 & GATA22F11 & Tetra & + & $200-250$ & 13 & 0.95 & 0.89 & 0.88 & 0.87 \\
\hline & 53 & D1S549 & GATA4H09 & Tetra & & 167-191 & 7 & 0.89 & 0.77 & 0.81 & 0.77 \\
\hline \multirow{9}{*}{12} & & D16S764 & GATA42E11 & Tetra & & 98-110 & 4 & 0.76 & 0.58 & 0.56 & 0.70 \\
\hline & 54 & D4S2366 & GATA22G05 & Tetra & & $116-136$ & 6 & 0.90 & 0.80 & 0.69 & 0.79 \\
\hline & & D16S539 & GATA11C06 & Tetra & & $146-162$ & 5 & 0.88 & 0.80 & 0.75 & 0.76 \\
\hline & 55 & D2S1328 & GATA27A12 & Tetra & & $134-158$ & 7 & 0.79 & 0.64 & 0.56 & 0.75 \\
\hline & & D3S4545 & GATA164B08 & Tetra & & $200-236$ & 6 & 0.86 & 0.72 & 0.66 & 0.82 \\
\hline & 56 & D12S372 & GATA4H03 & Tetra & & $172-188$ & 5 & 0.86 & 0.73 & 0.75 & 0.76 \\
\hline & & $\mathrm{D} 2 \mathrm{~S} 2976$ & GATA165C07 & Tetra & + & $194-228$ & 10 & 0.74 & 0.48 & 0.53 & 0.85 \\
\hline & 57 & $\mathrm{PAH}$ & $\mathrm{PAH}$ & Tetra & & $226-250$ & 6 & 0.86 & 0.71 & 0.59 & 0.80 \\
\hline & & D10S1435 & GATA88F09 & Tetra & & $251-271$ & 6 & 0.86 & 0.74 & 0.75 & 0.75 \\
\hline \multirow{9}{*}{13} & & D8S373 & UT721 & Tetra & & $190-222$ & 8 & 0.94 & 0.83 & 0.72 & 0.78 \\
\hline & 58 & D8S1113 & GGAA8G07 & Tetra & & $212-232$ & 6 & 0.80 & 0.65 & 0.56 & 0.81 \\
\hline & & DXS7132 & GATA72E05 & Tetra & & $280-296$ & 5 & & & & \\
\hline & 59 & D18S877 & GATA64H04 & Tetra & & $113-133$ & 6 & 0.86 & 0.70 & 0.69 & 0.68 \\
\hline & 60 & D1S1588* & ATA2E04 & Tri & & $113-134$ & 4 & 0.74 & 0.58 & 0.38 & 0.68 \\
\hline & 61 & D21S2055 & GATA188F04 & Tetra & & $115-203$ & 12 & 0.95 & 0.85 & 0.88 & 0.88 \\
\hline & 62 & DXS6814 & GGAT3F08 & Tetra & & $160-180$ & 4 & & & & \\
\hline & 63 & D2S1363 & GATA23D03 & Tetra & & $166-206$ & 8 & 0.84 & 0.70 & 0.81 & 0.79 \\
\hline & 64 & D9S922 & GATA21F05 & Tetra & + & $256-272$ & 6 & 0.87 & 0.75 & 0.75 & 0.78 \\
\hline \multirow{6}{*}{14} & & & GATA172D05 & Tetra & & $106-126$ & 5 & & & & \\
\hline & 65 & D6S1017 & GGAT3H10 & Tetra & & $154-178$ & 4 & 0.77 & 0.69 & 0.75 & 0.68 \\
\hline & & D10S1225 & ATA24F10 & Tri & & 179-191 & 5 & 0.85 & 0.74 & 0.75 & 0.76 \\
\hline & & D12S375 & GATA3F02 & Tetra & & $163-183$ & 6 & 0.86 & 0.77 & 0.88 & 0.74 \\
\hline & 66 & D4S1625 & GATA107 & Tetra & & $191-203$ & 4 & 0.81 & 0.66 & 0.66 & 0.74 \\
\hline & & $\mathrm{D} 2 \mathrm{~S} 2972$ & GATA176C01 & Tetra & + & $217-237$ & 7 & 0.89 & 0.76 & 0.66 & 0.77 \\
\hline
\end{tabular}


Table 1. Continued

\begin{tabular}{|c|c|c|c|c|c|c|c|c|c|c|c|}
\hline $\begin{array}{l}\text { Loading } \\
\text { Panel }\end{array}$ & $\begin{array}{l}\text { PCR } \\
\text { set }\end{array}$ & Locus & Marker & $\begin{array}{l}\text { Repeat } \\
\text { unit }\end{array}$ & $\begin{array}{l}\text { Irregular } \\
\text { allele }\end{array}$ & $\begin{array}{l}\text { Size } \\
\text { range }\end{array}$ & Allele & PD & $\begin{array}{l}\text { Expected } \\
\text { H. }\end{array}$ & $\begin{array}{l}\text { Observed } \\
\text { H. }\end{array}$ & $\begin{array}{l}\text { Observed H. } \\
\text { (Caucasian) }\end{array}$ \\
\hline & 67 & D12S395 & GATA4H01 & Tetra & + & $222-246$ & 9 & 0.87 & 0.79 & 0.78 & 0.76 \\
\hline & & D6S1277 & GATA81B01 & Tetra & & 281-309 & 6 & 0.84 & 0.72 & 0.81 & 0.72 \\
\hline & 68 & D10S1248 & GGAA23C05 & Tetra & & $238-258$ & 6 & 0.91 & 0.80 & 0.72 & 0.75 \\
\hline \multirow{9}{*}{15} & 69 & D7S1819 & GATA24F03 & Tetra & & $163-187$ & 7 & 0.86 & 0.76 & 0.63 & 0.73 \\
\hline & & D13S894 & GATA86H01 & Tetra & & 186-198 & 4 & 0.78 & 0.59 & 0.63 & 0.64 \\
\hline & 70 & D16S3253 & GATA22F09 & Tetra & & $166-198$ & 7 & 0.87 & 0.75 & 0.84 & 0.71 \\
\hline & & D15S657 & GATA22F01 & Tetra & & $329-353$ & 7 & 0.92 & 0.85 & 0.88 & 0.82 \\
\hline & 71 & D10S2325 & GAAT5F06 & Penta & & $110-155$ & 9 & 0.93 & 0.84 & 0.91 & 0.85 \\
\hline & 72 & D3S1744 & GATA3C02 & Tetra & & $133-165$ & 9 & 0.91 & 0.82 & 0.69 & 0.80 \\
\hline & 73 & D14S592 & ATA19H08 & Tri & & $216-240$ & 9 & 0.90 & 0.82 & 0.84 & 0.68 \\
\hline & 74 & D6S2439 & GATA163B10 & Tetra & & $222-250$ & 7 & 0.90 & 0.79 & 0.75 & 0.87 \\
\hline & 75 & D14S1280 & GATA31B09 & Tetra & & $281-297$ & 5 & 0.85 & 0.71 & 0.66 & 0.70 \\
\hline \multirow{6}{*}{16} & 76 & D12S1064 & GATA63D12 & Tetra & & 170-202 & 9 & 0.88 & 0.78 & 0.75 & 0.82 \\
\hline & & D1S534 & GATA12A07 & Tetra & + & $194-218$ & 9 & 0.90 & 0.77 & 0.81 & 0.83 \\
\hline & 77 & DXYS154 & & $\mathrm{Di}$ & & $225-251$ & 10 & & & & \\
\hline & & D4S2431 & GGAA19H07 & Tetra & + & $224-264$ & 12 & 0.87 & 0.76 & 0.78 & 0.82 \\
\hline & 78 & D17S928 & AFM217yd10 & Di & & $131-159$ & 14 & 0.94 & 0.87 & 0.84 & 0.79 \\
\hline & 79 & D21S1446 & GATA70B08 & Tetra & + & $206-224$ & 5 & 0.81 & 0.64 & 0.59 & 0.69 \\
\hline \multirow{9}{*}{17} & 80 & D1S1612 & GGAA3A07 & Tetra & & $102-130$ & 8 & 0.89 & 0.80 & 0.81 & 0.83 \\
\hline & & D4S1652 & GATA5B02 & Tetra & & $133-145$ & 4 & 0.78 & 0.58 & 0.56 & 0.82 \\
\hline & 81 & D17S1301 & GATA28D11 & Tetra & & 139-159 & 6 & 0.83 & 0.69 & 0.69 & 0.65 \\
\hline & & D19S433 & GGAA2A03 & Tetra & + & $195-213$ & 10 & 0.90 & 0.78 & 0.69 & 0.77 \\
\hline & 82 & D8S1119 & ATA19G07 & Tri & & 171-191 & 7 & 0.88 & 0.80 & 0.78 & 0.80 \\
\hline & & D9S925 & GATA27A11 & Tetra & & 180-196 & 5 & 0.90 & 0.79 & 0.72 & 0.82 \\
\hline & 83 & D10S1432 & GATA87G01 & Tetra & + & $159-179$ & 7 & 0.88 & 0.74 & 0.66 & 0.74 \\
\hline & 84 & D3S2390 & GATA31E08 & Tetra & & $227-251$ & 7 & & & & \\
\hline & 85 & D7S3046 & GATA118G10 & Tetra & & $321-349$ & 8 & 0.92 & 0.82 & 0.81 & 0.81 \\
\hline \multirow{9}{*}{18} & & D10S1426 & GATA73E11 & Tetra & & $154-174$ & 5 & 0.83 & 0.70 & 0.66 & 0.74 \\
\hline & 86 & & GATA184A08 & Tetra & & $168-200$ & 9 & 0.92 & 0.86 & 0.91 & 0.78 \\
\hline & & D1S518 & GATA7C01 & Tetra & & $188-212$ & 7 & 0.91 & 0.78 & 0.66 & 0.84 \\
\hline & 87 & D10S1213 & GGAA5D10 & Tetra & & 85-129 & 10 & 0.88 & 0.72 & 0.81 & 0.80 \\
\hline & & D6S1053 & GATA64D02 & Tetra & & $294-314$ & 6 & 0.90 & 0.80 & 0.75 & 0.81 \\
\hline & 88 & D12S1042 & ATA27A06 & Tri & & $114-132$ & 7 & 0.93 & 0.83 & 0.75 & 0.81 \\
\hline & 89 & D8S1132 & GATA26E03 & Tetra & & $138-166$ & 8 & 0.93 & 0.87 & 0.88 & 0.86 \\
\hline & 90 & D3S1766 & GATA6F06 & Tetra & & $212-232$ & 6 & 0.90 & 0.78 & 0.78 & 0.76 \\
\hline & 91 & D4S2394 & ATA26B08 & Tri & & $233-251$ & 5 & 0.76 & 0.63 & 0.75 & 0.79 \\
\hline \multirow{9}{*}{19} & & D8S1106 & GATA23D06 & Tetra & & $134-146$ & 4 & 0.83 & 0.68 & 0.75 & 0.73 \\
\hline & 92 & D13S796 & GATA51B02 & Tetra & & $146-166$ & 6 & 0.89 & 0.79 & 0.78 & 0.80 \\
\hline & & D12S391 & GATA11H08 & Tetra & & $207-247$ & 11 & 0.93 & 0.86 & 0.88 & 0.88 \\
\hline & 93 & D13S317 & GATA7G10 & Tetra & & $175-195$ & 6 & 0.90 & 0.82 & 0.91 & 0.79 \\
\hline & & D9S2169 & GATA62F03 & Tetra & & $274-290$ & 5 & 0.83 & 0.68 & 0.66 & 0.64 \\
\hline & 94 & D9S910 & ATA18A07 & Tri & & $101-125$ & 6 & 0.82 & 0.68 & 0.59 & 0.66 \\
\hline & 95 & D3S4529 & GATA128C02 & Tetra & & $147-163$ & 5 & 0.88 & 0.78 & 0.81 & 0.72 \\
\hline & 96 & D8S1477 & GGAA20C10 & Tetra & & $157-189$ & 7 & 0.92 & 0.81 & 0.69 & 0.86 \\
\hline & 97 & D13S787 & GATA23C03 & Tetra & & $247-263$ & 5 & 0.77 & 0.61 & 0.44 & 0.72 \\
\hline \multirow{8}{*}{20} & & D6S1027 & ATA22G07 & Tri & & $110-134$ & 7 & 0.83 & 0.70 & 0.53 & 0.77 \\
\hline & 98 & D16S2624 & GATA81D12 & Tetra & & $127-147$ & 6 & 0.81 & 0.65 & 0.59 & 0.70 \\
\hline & & D20S482 & GATA51D03 & Tetra & & $140-164$ & 7 & 0.87 & 0.73 & 0.59 & 0.68 \\
\hline & 99 & D17S1290 & GATA49C09 & Tetra & & $172-208$ & 8 & 0.88 & 0.80 & 0.88 & 0.84 \\
\hline & 100 & D2S2968 & GATA178G09 & Tetra & & $177-189$ & 4 & 0.64 & 0.48 & 0.41 & 0.72 \\
\hline & 101 & D18S844 & ATA1H06 & Tri & & 177-198 & 6 & 0.87 & 0.76 & 0.81 & 0.76 \\
\hline & 102 & DXS6810 & GATA69C12 & Tetra & & $214-238$ & 5 & & & & \\
\hline & 103 & D13S793 & GATA43H03 & Tetra & & $248-272$ & 7 & 0.89 & 0.80 & 0.69 & 0.77 \\
\hline
\end{tabular}

*Significant deviations $(P<0.05)$ from Hardy-Weinberg equilibrium $(\mathrm{HWE})$ were observed in all three tests used (see text for details) $\mathrm{PD}$, Power of discrimination; $\mathrm{H}$, heterozygosity 
Fig. 1. Distribution of expected and observed heterozygosities (Exp. $H$ [black bars] and Obs. $H$ [white bars]) in the Japanese population and the observed heterozygosity (Obs. $H$ [gray bars]) in the Caucasian population

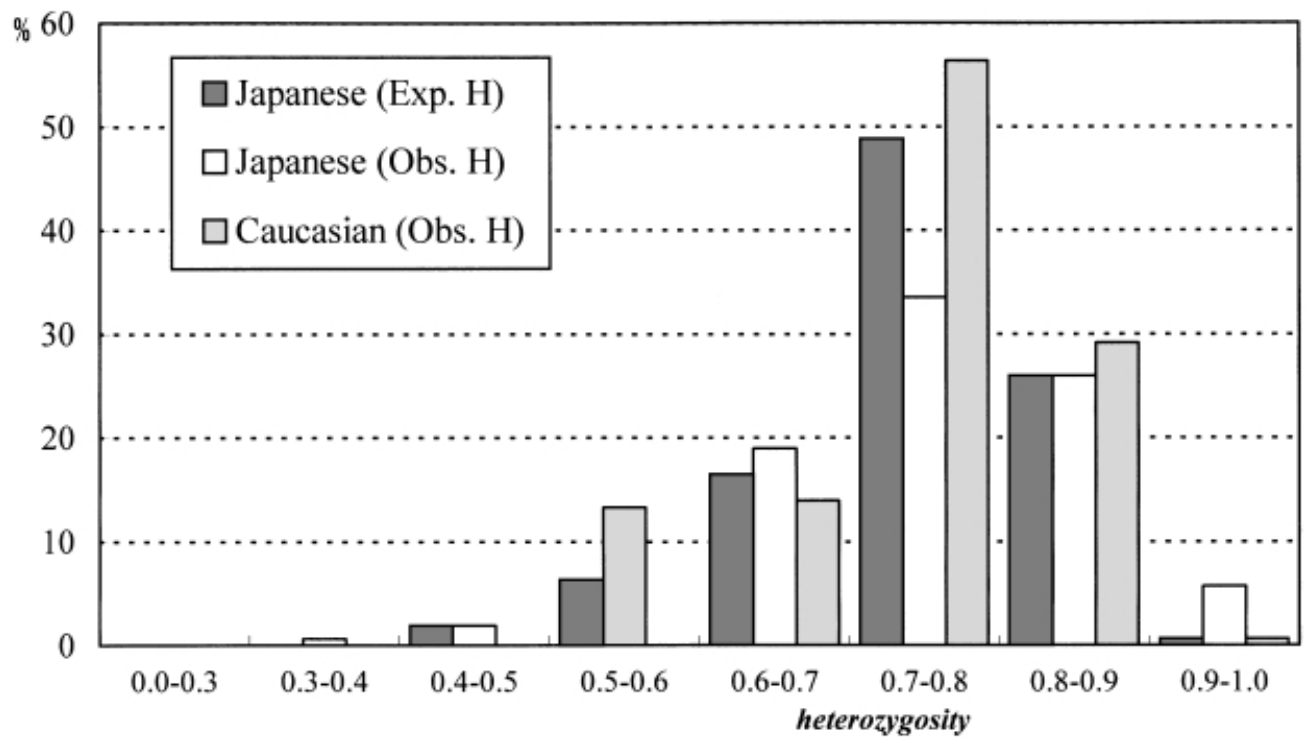

b)

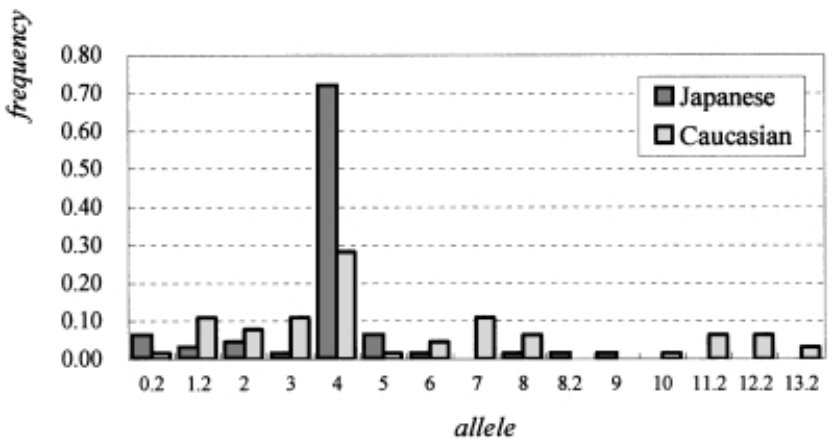

d)

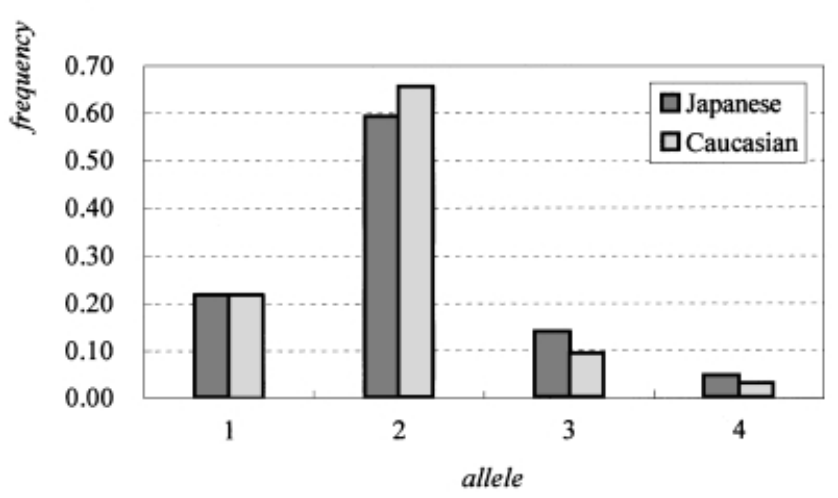

Fig. 2a-d. Examples of allele distributions in representative loci in the Japanese (black bars) and Caucasian (gray bars) populations. a At D7S1823; b at D2S2976; $\mathbf{c}$ at GATA184A08; $\mathbf{d}$ at D13S894

than in Caucasians. The most frequent allele, allele 4, at D2S2976 reached a frequency of $72 \%$ in Japanese, but the frequency was only $28 \%$ in Caucasians. The heterozygosity at this locus was only 0.53 in Japanese, against 0.84 in Caucasians. At the GATA184A08 marker, the Japanese alleles were distributed more equally than the Caucasian alleles.
On the other hand, D13S894 showed very similar allelic distributions in both populations. At the three loci D7S1823, D2S2976, and GATA184A08, significant differences $(P<0.05)$ were observed between Japanese and Caucasians, while no significant differences were seen at the D13S894 locus. 
We divided 130 autosomal tetranucleotide STRs in the screening set into two groups; those with and those without irregular alleles. One group consisted of 19 loci with irregular alleles found in this study, and the other group consisted of the other 111 loci, without irregular alleles. Comparison of the allele frequency distributions (Table 2) revealed that most of the loci with irregular alleles $(79 \%)$ had two or more peaks of allele frequency distribution, while most of the loci without irregular alleles $(76 \%)$ showed single-peak allele frequency distribution. The number of alleles per
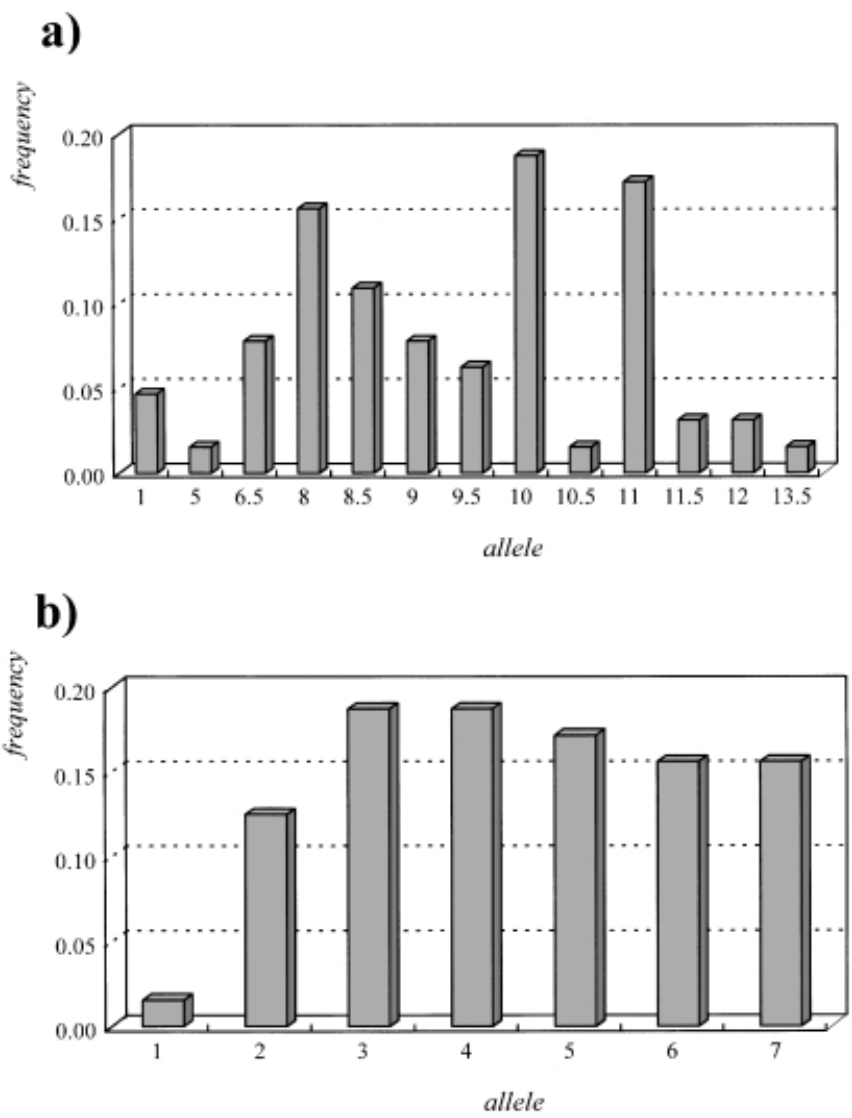

Fig. 3a,b. Typical examples of allele distribution in loci with and without irregular alleles, a at D3S2427 and b at D6S1056, in the Japanese population. The Exp. H, Obs. H, and power of discrimination values were $0.89,0.88$, and 0.95 , respectively, for D3S2427; and 0.85, 0.94, and 0.92, respectively, for D6S1056

Table 2. Correlation between irregular alleles and properties of markers

\begin{tabular}{llc}
\hline Irregular allele & With & Without \\
\hline Number of markers & 19 & 111 \\
Bimodal markers $(\%)^{\mathrm{a}}$ & 79 & 24 \\
Mean number of alleles per locus $_{\text {Observed heterozygosities }}$ & 9.47 & 6.62 \\
Expected heterozygosities & 0.732 & 0.732 \\
Power of discrimination & 0.763 & 0.745 \\
Departure from HWE $(\%)^{\mathrm{b}}$ & 0.880 & 0.864 \\
\hline
\end{tabular}

${ }^{a}$ Proportion of loci with bimodal allele distribution

${ }^{\mathrm{b}}$ Proportion of loci with $P$ value of less than 0.05 in the three tests for HWE locus was also larger in loci with irregular alleles (9.5) than in loci without irregular alleles (6.6). There were, however, no significant differences among observed heterozygosities, expected heterozygosities, and powers of discrimination between the two groups. Figure 3 shows a typical example of the allele distribution locus in each group. Although the number of alleles at D3S2427 showing bimodal distribution was about twice more than that at D6S1056, showing almost equal allele frequency at each allele except allele 1 , both loci had very high observed heterozygosities (0.88 at D3S2427 and 0.94 at D6S1056). Also, no significant differences concerning the proportion of loci departing from HWE were found between STR loci with and without irregular alleles. Therefore, STR loci without irregular alleles are preferable for use in practice because of their simplicity and convenience in typing.

The present study provides basic data for investigating linkage analyses in genetic diseases and for use in forensic practice (e.g., in personal identification and paternity tests). Consequently, this database would be useful for selecting STR loci suitable for forensic applications in the Japanese population, and for constructing an optimal multiplex STR typing system. Furthermore, a future comparative study of these STR loci in Japanese and other ethnic populations would give precise information about their genetic relationships.

Acknowledgments We are grateful to Dr. Yuri E. Dubrova for providing Caucasian DNA samples. This work was supported in part by Grants-in-Aid for Scientific Research from the Ministry of Education, Science, Sports, and Culture of Japan.

\section{References}

Bowcock AM, Ruiz-Linares A, Tomfohrde J, Minch E, Kidd JR, Cavalli-Sforza LL (1994) High resolution of human evolutionary trees with polymorphic microsatellites. Nature 368:455-457

Chakraborty R, Fornage M, Gueguen R, Boerwinkle E (1991) Population genetics of hypervariable loci: analysis of PCR based VNTR polymorphism within a population. In: Burke T, Dolf G, Jeffreys AJ, Wolff R (eds) DNA fingerprinting: approaches and applications. Birkhauser Verlag, Berlin, pp 127-143

Dubovsky J, Sheffield VC, Duyk GM, Weber JL (1995) Sets of short tandem repeat polymorphisms for efficient linkage screening of the human genome. Hum Mol Genet 4:449-452

Edwards A, Hammond HA, Jin L, Caskey CT, Chakraborty R (1992) Genetic variation at five trimeric and tetrameric tandem repeat loci in four human population groups. Genomics 12:241-253

Fisher RA (1951) Standard calculations for evaluating a blood-group system. Heredity 5:95-102

Gill P, Brinkmann B, d'Aloja E, Anderson J, Bar W, Carracedo A, Dupuy B, Eriksen B, Jangblad M, Johnsson V, Kloosterman AD, Lincoln P, Morling N, Rand S, Sabatier M, Scheithauer R, Schneider P, Vide MC (1997) Considerations from the European DNA profiling group (EDNAP) concerning STR nomenclature. Forensic Sci Int 87:185-192

Guo SW, Thompson EA (1992) Performing the exact test of HardyWeinberg proportion for multiple alleles. Biometrics 48:361-372

Gyapay G, Morissette J, Vignal A, Dib C, Fizames C, Millasseau P, Marc S, Bernardi G, Lathrop M, Weissenbach J (1994) The 1993-94 Genethon human genetic linkage map. Nat Genet 7:246-339

International Human Genome Sequencing Consortium (2001) Initial sequencing and analysis of the human genome. Nature 409:860921 
Micka KA, Sprecher CJ, Lins AM, Theisen Comey C, Koons BW, Crouse C, Endean D, Pirelli K, Lee SB, Duda N, Ma M, Schumm JW (1996) Validation of multiplex polymorphic STR amplification sets developed for personal identification applications. J Forensic Sci 41:582-590

Raymond M, Rousset F (1995) GENEPOP (version 1.2): population genetics software for exact tests and ecumenicism. J Hered 86:248249

Schellenberg GD, Bird TD, Wijsman EM, Orr HT, Anderson L, Nemwns E, White JA, Bonnycastle L, Weber JL, Alonso E, Potter H, Heston LL, Martin GM (1992) Genetic linkage evidence for a familial Alzheimer's disease locus on chromosome 14. Science 258:668-671

Siegel S (1956) Nonparametric statistics for the behavioral sciences. McGraw-Hill, New York

Sweet D, Hildebrand D (1999) Saliva from cheese bite yields DNA profile of burglar: a case report. Int J Legal Med 112:201-203
Tamaki K, Yamamoto T, Uchihi R, Katsumata Y, Kondo K, Mizuno S, Kimura A, Sasazuki T (1991) Frequency of HLA-DQA1 alleles in the Japanese population. Hum Hered 41:209-214

Thomson JA, Pilotti V, Stevens P, Ayres KL, Debenham PG (1999) Validation of short tandem repeat analysis for the investigation of cases of disputed paternity. Forensic Sci Int 100:1-16

Weir BS (1992) Independence of VNTR alleles defined as fixed bins. Genetics 130:873-887

Wijmenga C, Frants RR, Brouwer OF, Moerer P, Weber JL, Padberg GW (1990) Location of facioscapulohumeral muscular dystrophy gene on chromosome 4. Lancet 336:651-653

Yamamoto T, Uchihi R, Nozawa H, Huang XL, Leong YK, Tanaka M, Mizutani M, Tamaki K, Katsumata Y (1999) Allele distribution at nine STR loci - D3S1358, vWA, FGA, TH01, TPOX, CSF1PO, D5S818, D13S317 and D7S820 - in the Japanese population by multiplex PCR and capillary electrophoresis. J Forensic Sci 44:167170 